\title{
Spring Cell Equivalence of Simplex Finite Elements - Exploration of an Iterative Approach
}

\author{
IOANNIS DOLTSINIS \\ Institute of Statics and Dynamics of Aerospace Structures \\ Faculty of Aerospace Engineering and Geodesy \\ University of Stuttgart \\ Pfaffenwaldring 27, 70569 Stuttgart \\ GERMANY
}

\begin{abstract}
Natural spring cell substitutes of triangular and tetrahedral finite elements at constant strain take advantage of a formalism oriented along the element sides/edges. Two different models in use account just for the diagonal entities of either the flexibility matrix of the element or of its stiffness matrix. Both are incomplete substitutes, and defective to a degree depending on the significance of the off-diagonal parts of the element matrices. The present work discusses an iterative completeness of the substitution accounting for the discarded parts by additives to the spring members of the cell. In this connection, the iteration schemes are set up for either model at the material and at the element level, and convergence criteria are defined in terms of the spectral radii of the iteration operators. The convergence regions are confined for triangular elements, and are demonstrated with reference to a casestudy.
\end{abstract}

Key-Words: Spring cells, finite elements, equivalent substitution, iterative approach, convergence criteria.

Received: August 11, 2020. Revised: October 29, 2020. Accepted: November 6, 2020. Published: November 14, 2020.

\section{Introduction}

The natural setup of simplex finite elements, the triangle and the tetrahedron at constant strain, refers to static and kinematic quantities defined along the sides and the edges of the element [1]. A simple transition to the spring cell assigns either the diagonal entities of the flexibility matrix of the elastic element or those of the stiffness matrix to the springs, or bar members, that build the cell [2], [3]. Both approaches lead to defective substitutes of the finite element except for the ideal constellation of the regular triangle of a material with the coefficient of lateral contraction $\nu=1 / 3$. A certain superiority of the flexibility concept experienced in computations $[4,5]$ has been supported also theoretically [6]. Beyond isotropic elasticity, anisotropy is discussed in [3] and the implication of plasticity in [5], [7].

Other cell models assembled of pin-joined bars prove similarly deficient [8]. Complete representation of the continuum finite element by additional variables $[9,10]$ deviates from the pin-joined bar concept. The present account discusses completeness of the substitution without leaving the concept of the pin-joined bars. Thereby the bars are enriched by additional displacements or forces which account for the discarded off-diagonal parts of the flexibility- and the stiffness matrix of the finite element. The result- ing implicit forms are interpreted as iterative solution schemes for force and displacement. Their convergence properties are investigated at the element level and at the local, constitutive level of stress and strain. In this connection, convergence criteria are defined for the iteration at given stress, and at given strain, which may be associated with the limiting cases of either static or kinematic determinacy of the structure.

Details are elaborated for triangular configurations. Thereby the range of convergence is shown as a function of the element shape and allows an assessment of the different iteration schemes. The theoretical exposition is complemented by a numerical example which demonstrates the relevance of the formal prediction. The last step is to the structural assembly. As a rule the finite element solution is for the displacement, which is attempted by iteration of the complete spring cell substitute. A sufficient condition for convergence brings back to the element level and indicates an inferiority of the flexibility cell due to the displacement based solution algorithm for the system.

Section 2 recalls the transition from the natural finite element to the spring cell, introduces the splitting of the element matrices in diagonal- and off-diagonal parts and defines the cell supplements that complete the substitution of the finite element. Section 3 is concerned with the iterative solution in the stiffness con- 


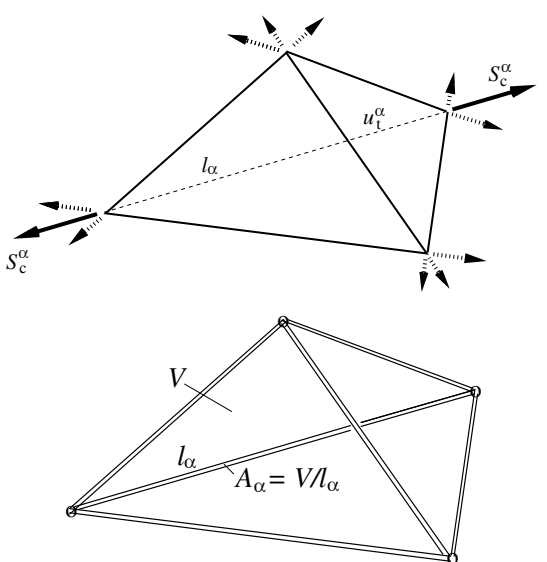

Figure 1: Tetrahedral finite element and skeletal spring cell. Definition of natural quantities.

text and defines convergence criteria at the elementand at the material level, while Section 4 deals with the subject from the flexibility point of view. Section 5 focuses on triangular configurations and estimates the range of convergence in dependence of the shape for each one of the iterative schemes, and Section 6 demonstrates the procedures by a numerical example. Section 7 is concerned with the discretized structure regarding the iterative solution for the displacements and the assessment of convergence. Section 8 summarizes the essential issues of the account and points out the main results.

\section{From the natural finite element to the spring cell}

The finite elements considered in the present context are the constant strain tetrahedron and the plane triangle [1]. The lines connecting nodal points specify the natural directions where relevant quantities are referred to (Fig 1). Quantities defined along the natural directions are denoted either total or component. The lengths of the connecting lines are arranged as the diagonal matrix $\mathbf{l}=\left\lceil l_{\vartheta}\right\rfloor$, triangle: $\vartheta=\alpha, \beta, \gamma$ tetrahedron: $\vartheta=\alpha, \cdots, \zeta$. Elongations define the total displacements collected in the vector array $\mathbf{u}_{\mathrm{t}}=\left\{u_{\mathrm{t}}^{\vartheta}\right\}$, the unit elongations are the total strains in $\varepsilon_{\mathrm{t}}=\left\{\varepsilon_{\mathrm{t}}^{\vartheta}\right\}$ such that

$$
\varepsilon_{\mathrm{t}}=\mathbf{l}^{-1} \mathbf{u}_{\mathrm{t}} \text {. }
$$

The corresponding forces acting at the nodal points along the natural directions are collected in the vector array $\mathbf{S}_{\mathrm{c}}=\left\{S_{\mathrm{c}}^{\vartheta}\right\}$. They result from imposed component stresses in $\boldsymbol{\sigma}_{\mathrm{c}}=\left\{\sigma_{\mathrm{c}}^{\vartheta}\right\}$ and vice versa. Work equivalence relates force and stress

$$
\mathbf{S}_{\mathrm{c}}=V \mathbf{1}^{-1} \boldsymbol{\sigma}_{\mathrm{c}} \Leftarrow \mathbf{A} \boldsymbol{\sigma}_{\mathrm{c}} .
$$

The first expression refers to the finite element of volume $V$. The transition to the second expression leads to a cell built of bars between the nodal points. These carry individually the stress $\sigma_{\mathrm{c}}$ that acts on the crosssection areas defined in the diagonal matrix

$$
\mathbf{A}=\left\lceil A_{\vartheta}\right\rfloor=\left\lceil\frac{V}{l_{\vartheta}}\right\rfloor=V \mathbf{l}^{-1} .
$$

In linear elasticity the relationship between the stress $\sigma_{\mathrm{c}}$ and the strain $\varepsilon_{\mathrm{t}}$ is stated as

$$
\sigma_{\mathrm{c}}=\kappa_{\mathrm{N}} \varepsilon_{\mathrm{t}}
$$

where $\kappa_{\mathrm{N}}$ is denoted the natural stiffness matrix of the elastic material. With this eqn (2) for the finite element becomes

$$
\mathbf{S}_{\mathrm{c}}=\mathbf{k}_{\mathrm{N}} \mathbf{u}_{\mathrm{t}} \Leftarrow \mathbf{k}_{\mathrm{D}} \mathbf{u}_{\mathrm{t}}+\mathbf{J}_{\mathrm{k}},
$$

where the stiffness matrix of the element

$$
\mathbf{k}_{\mathrm{N}}=V \mathbf{l}^{-1} \boldsymbol{\kappa}_{\mathrm{N}} \mathbf{l}^{-1} \Leftarrow \mathbf{k}_{\mathrm{D}}+\mathbf{k}_{-},
$$

has been partitioned such that $\mathbf{k}_{\mathrm{D}}$ refers to the diagonal of the element stiffness matrix. The part $\mathbf{k}_{-}=$ $\mathbf{k}_{\mathrm{N}}-\mathbf{k}_{\mathrm{D}}$ denotes the remaining off-diagonal matrix which in eqn (5) gives rise to the term

$$
\mathbf{J}_{\mathrm{k}}=\mathbf{k}_{-} \mathbf{u}_{\mathrm{t}} .
$$

The transition to the cell as indicated on the right-hand side of eqn (5) is for elastic bars pin-joined at the nodal points. Their elastic response is described by the diagonal $\mathrm{k}_{\mathrm{D}}$ of the element stiffness matrix, while the off-diagonal terms of the matrix are accounted for by the supplementary forces $\mathbf{J}_{\mathrm{k}}$.

The natural flexibility matrix $\phi_{\mathrm{N}}$ of the elastic material relates the strain $\varepsilon_{\mathrm{t}}$ with the stress $\sigma_{\mathrm{c}}$.

$$
\varepsilon_{\mathrm{t}}=\phi_{\mathrm{N}} \sigma_{\mathrm{c}} .
$$

Substitution in eqn (1) and with the stress from eqn (2), gives the elongations in terms of forces

$$
\mathbf{u}_{\mathrm{t}}=\mathbf{f}_{\mathrm{N}} \mathbf{S}_{\mathrm{c}} \Leftarrow \mathbf{f}_{\mathrm{D}} \mathbf{S}_{\mathrm{c}}+\mathbf{h}_{\mathrm{f}},
$$

where the flexibility matrix of the element has been partitioned for the transition to the cell

$$
\mathbf{f}_{\mathrm{N}}=\frac{1}{V} \mathbf{l} \phi_{\mathrm{N}} \mathbf{l} \Leftarrow \mathbf{f}_{\mathrm{D}}+\mathbf{f}_{-} .
$$

The first expression in eqn (9) refers to the finite element. In the cell form on the right-hand side the diagonal of the flexibility matrix relates the elongations of the bar members of the cell with the forces, and the supplement

$$
\mathbf{h}_{\mathrm{f}}=\mathbf{f}_{-} \mathbf{S}_{\mathrm{c}},
$$


accounts for the off-diagonal part of the matrix, which completes the substitution.

Equation (5) introduces the stiffness approach to the spring cell, and eqn (9) introduces the flexibility approach. The symbolism applies to the two- and the three-dimensional element with the content adapted. As long as the transition to the spring cell is based on the diagonal of the element matrices, either concept offers an incomplete substitution [2]. The following discusses completeness by an iterative incorporation of the off-diagonal parts of the element matrices.

\section{Iterative stiffness method}

\subsection{The finite element cycle}

The partitioned eqn (5) substitutes the finite element by a cell assembled of elastic bars pin-joined at the nodal points. The elastic bars cover the diagonal $\mathbf{k}_{\mathrm{D}}$ of the element stiffness matrix, and supplementary forces $\mathbf{J}_{\mathrm{k}}=\mathbf{k}_{-} \mathbf{u}_{\mathrm{t}}$ complete the substitution.

In the finite element statement of the structural problem, both the element forces and the displacements are unknown quantities. In the following, two limiting cases will treated on the element and on the local material level: the case of prescribed displacement and the case of imposed stress. If the displacement $\mathbf{u}_{\mathrm{t}}$ is prescribed, eqn (5) can be evaluated for the force. In case that the force $\mathbf{S}_{\mathrm{c}}$ is imposed, on the other hand, the cell variant of eqn (5) along with eqn (7) for the supplement enter an iterative solution scheme for the displacement

$$
\mathbf{u}_{\mathrm{t}, i+1}=\mathbf{k}_{\mathrm{D}}^{-1}\left(\mathbf{S}_{\mathrm{c}}-\mathbf{k}_{-} \mathbf{u}_{\mathrm{t}, i}\right) .
$$

Regarding consecutive iteration cycles

$$
\left(\delta \mathbf{u}_{\mathrm{t}}\right)_{i+1}=-\mathbf{k}_{\mathrm{D}}^{-1} \mathbf{k}_{-}\left(\delta \mathbf{u}_{\mathrm{t}}\right)_{i},
$$

where

$$
\begin{aligned}
\left(\delta \mathbf{u}_{\mathrm{t}}\right)_{i+1} & =\mathbf{u}_{\mathrm{t}, i+1}-\mathbf{u}_{\mathrm{t}, i} \\
\left(\delta \mathbf{u}_{\mathrm{t}}\right)_{i} & =\mathbf{u}_{\mathrm{t}, i}-\mathbf{u}_{\mathrm{t}, i-1} .
\end{aligned}
$$

Taking norms,

$$
\begin{aligned}
\left\|\left(\delta \mathbf{u}_{\mathrm{t}}\right)_{i+1}\right\| & =\left\|\mathbf{k}_{\mathrm{D}}^{-1} \mathbf{k}_{-}\left(\delta \mathbf{u}_{\mathrm{t}}\right)_{i}\right\| \\
& \leq\left\|\mathbf{k}_{\mathrm{D}}^{-1} \mathbf{k}_{-}\right\|\left\|\left(\delta \mathbf{u}_{\mathrm{t}}\right)_{i}\right\|,
\end{aligned}
$$

which suggests to state the convergence condition as

$$
\begin{aligned}
\omega_{\mathrm{ku}} & =\left\|\mathbf{k}_{\mathrm{D}}^{-1} \mathbf{k}_{-}\right\| \\
& =\max \sqrt{\lambda\left[\left(\mathbf{k}_{\mathrm{D}}^{-1} \mathbf{k}_{-}\right)^{\mathrm{t}}\left(\mathbf{k}_{\mathrm{D}}^{-1} \mathbf{k}_{-}\right)\right]}<1,
\end{aligned}
$$

where $\lambda[\cdots]$ denotes eigenvalues of the matrix product within the parentheses.

The above defines the spectral norm $\omega_{\mathrm{ku}}$, the maximum singular value of the iteration matrix in eqn (13) as a criterion for convergence. This quantity will serve as a convergence indicator.

The force supplement, eqn (7), can be expressed in terms of the stress resultants $\mathbf{S}_{\mathrm{c}}$ using eqn (5) for the finite element

$$
\mathbf{J}_{\mathrm{k}}=\mathbf{k}_{-} \mathbf{k}_{\mathrm{N}}^{-1} \mathbf{S}_{\mathrm{c}} .
$$

Imposed force determines then the supplement and the displacement can be computed directly. In the case of prescribed displacement the solution for the unknown force $\mathbf{S}_{\mathrm{c}}$ in the cell is attempted iteratively

$$
\mathbf{S}_{\mathrm{c}, i+1}=\mathbf{k}_{\mathrm{D}} \mathbf{u}_{\mathrm{t}}+\mathbf{k}_{-} \mathbf{k}_{\mathrm{N}}^{-1} \mathbf{S}_{\mathrm{c}, i} .
$$

For consecutive iteration cycles

$$
\left(\delta \mathbf{S}_{\mathrm{c}}\right)_{i+1}=\mathbf{k}_{-} \mathbf{k}_{\mathrm{N}}^{-1}\left(\delta \mathbf{S}_{\mathrm{c}}\right)_{i} .
$$

Norms are related as

$$
\begin{array}{r}
\left\|\left(\delta \mathbf{S}_{\mathrm{c}}\right)_{i+1}\right\|=\left\|\mathbf{k}_{-} \mathbf{k}_{\mathrm{N}}^{-1}\left(\delta \mathbf{S}_{\mathrm{c}}\right)_{i}\right\| \\
\leq\left\|\mathbf{k}_{-} \mathbf{k}_{\mathrm{N}}^{-1}\right\|\left\|\left(\delta \mathbf{S}_{\mathrm{c}}\right)_{i}\right\|,
\end{array}
$$

and for convergence

$$
\begin{aligned}
\omega_{\mathrm{kS}} & =\left\|\mathbf{k}_{-} \mathbf{k}_{\mathrm{N}}^{-1}\right\| \\
& =\max \sqrt{\lambda\left[\left(\mathbf{k}_{-} \mathbf{k}_{\mathrm{N}}^{-1}\right)^{\mathrm{t}}\left(\mathbf{k}_{-} \mathbf{k}_{\mathrm{N}}^{-1}\right)\right]}<1 .
\end{aligned}
$$

Schemes that are a direct consequence of the partitioning as in eqn (12) will be denoted primary, those deduced as in eqn (18) will be secondary.

\subsection{The local level}

Inspection of eqn (6) reveals that partitioning involves essentially the diagonal and off-diagonal constituents of the material stiffness matrix

$$
\kappa_{\mathrm{N}} \Leftarrow \kappa_{\mathrm{D}}+\kappa_{-} .
$$

Thereby the relationship between natural stress and strain transfers from the continuum to the cell members

$$
\sigma_{\mathrm{c}}=\kappa_{\mathrm{N}} \varepsilon_{\mathrm{t}} \Leftarrow \kappa_{\mathrm{D}} \varepsilon_{\mathrm{t}}+\tau_{\kappa} .
$$

The stress supplement

$$
\tau_{\kappa}=\kappa_{-} \varepsilon_{\mathrm{t}},
$$

depends on the strain which eventually determines the stress. If instead the stress is imposed, the cell variant in eqn (23) is solved iteratively for the strain:

$$
\varepsilon_{\mathrm{t}, i+1}=\boldsymbol{\kappa}_{\mathrm{D}}^{-1}\left(\boldsymbol{\sigma}_{\mathrm{c}}-\boldsymbol{\kappa}_{-} \varepsilon_{\mathrm{t}, i}\right) .
$$


Changes between consecutive cycles are related as

$$
\left(\delta \varepsilon_{\mathrm{t}}\right)_{i+1}=-\boldsymbol{\kappa}_{\mathrm{D}}^{-1} \boldsymbol{\kappa}_{-}\left(\delta \varepsilon_{\mathrm{t}}\right)_{i}
$$

Following previous arguments, convergence of norms is ensured if

$$
\begin{aligned}
\omega_{\kappa \varepsilon} & =\left\|\boldsymbol{\kappa}_{\mathrm{D}}^{-1} \boldsymbol{\kappa}_{-}\right\| \\
& =\max \sqrt{\lambda\left[\left(\boldsymbol{\kappa}_{\mathrm{D}}^{-1} \boldsymbol{\kappa}_{-}\right)^{\mathrm{t}}\left(\boldsymbol{\kappa}_{\mathrm{D}}^{-1} \boldsymbol{\kappa}_{-}\right)\right]}<1 .
\end{aligned}
$$

In case that the stress supplement in eqn (23) is expressed in the form

$$
\boldsymbol{\tau}_{\kappa}=\boldsymbol{\kappa}_{-} \boldsymbol{\kappa}_{\mathrm{N}}^{-1} \boldsymbol{\sigma}_{\mathrm{c}}
$$

knowledge of $\sigma_{\mathrm{c}}$ determines the strain in the cell context. At imposed strain the stress is approached iteratively

$$
\boldsymbol{\sigma}_{\mathrm{c}, i+1}=\boldsymbol{\kappa}_{\mathrm{D}} \varepsilon_{\mathrm{t}}+\boldsymbol{\kappa}_{-} \boldsymbol{\kappa}_{\mathrm{N}}^{-1} \boldsymbol{\sigma}_{\mathrm{c}, i}
$$

and

$$
\left(\delta \boldsymbol{\sigma}_{\mathrm{c}}\right)_{i+1}=\boldsymbol{\kappa}_{-} \boldsymbol{\kappa}_{\mathrm{N}}^{-1}\left(\delta \boldsymbol{\sigma}_{\mathrm{c}}\right)_{i} .
$$

The requirement for convergence is

$$
\begin{aligned}
\omega_{\kappa \sigma} & =\left\|\boldsymbol{\kappa}_{-} \boldsymbol{\kappa}_{\mathrm{N}}^{-1}\right\| \\
& =\max \sqrt{\lambda\left[\left(\boldsymbol{\kappa}_{-} \boldsymbol{\kappa}_{\mathrm{N}}^{-1}\right)^{\mathrm{t}}\left(\boldsymbol{\kappa}_{-} \boldsymbol{\kappa}_{\mathrm{N}}^{-1}\right)\right]}<1 .
\end{aligned}
$$

\section{Iterative flexibility method}

\subsection{The finite element cycle}

In the cell variant of eqn (9) imposed forces $\mathbf{S}_{c}$ determine the displacement $\mathbf{u}_{\mathrm{t}}$ while at prescribed displacements the forces are computed iteratively. In conjunction with eqn (11),

$$
\mathbf{S}_{\mathrm{c}, i+1}=\mathbf{f}_{\mathrm{D}}^{-1}\left(\mathbf{u}_{\mathrm{t}}-\mathbf{f}_{-} \mathbf{S}_{\mathrm{c}, i}\right) .
$$

For consecutive iteration cycles

$$
\left(\delta \mathbf{S}_{\mathrm{c}}\right)_{i+1}=-\mathbf{f}_{\mathrm{D}}^{-1} \mathbf{f}_{-}\left(\delta \mathbf{S}_{\mathrm{c}}\right)_{i} .
$$

The norm

$$
\begin{aligned}
\left\|\left(\delta \mathbf{S}_{\mathrm{c}}\right)_{i+1}\right\| & =\left\|\mathbf{f}_{\mathrm{D}}^{-1} \mathbf{f}_{-}\left(\delta \mathbf{S}_{\mathrm{c}}\right)_{i}\right\| \\
& \leq\left\|\mathbf{f}_{\mathrm{D}}^{-1} \mathbf{f}_{-}\right\|\left\|\left(\delta \mathbf{S}_{\mathrm{c}}\right)_{i}\right\|
\end{aligned}
$$

diminishes as long as

$$
\begin{aligned}
\omega_{\mathrm{fS}} & =\left\|\mathbf{f}_{\mathrm{D}}^{-1} \mathbf{f}_{-}\right\| \\
& =\max \sqrt{\lambda\left[\left(\mathbf{f}_{\mathrm{D}}^{-1} \mathbf{f}_{-}\right)^{\mathrm{t}}\left(\mathbf{f}_{\mathrm{D}}^{-1} \mathbf{f}_{-}\right)\right]}<1 .
\end{aligned}
$$

A secondary scheme is obtained by expressing the supplement, eqn (11), in terms of the displacement

$$
\mathbf{h}_{\mathrm{f}}=\mathbf{f}_{-} \mathbf{f}_{\mathrm{N}}^{-1} \mathbf{u}_{\mathrm{t}},
$$

which determines the force in the cell members. If the force is imposed eqn (9) is solved iteratively for the displacement

$$
\mathbf{u}_{\mathrm{t}, i+1}=\mathbf{f}_{\mathrm{D}} \mathbf{S}_{\mathrm{c}}+\mathbf{f}_{-} \mathbf{f}_{\mathrm{N}}^{-1} \mathbf{u}_{\mathrm{t}, i}
$$

and the difference between cycles progresses as

$$
\left(\delta \mathbf{u}_{\mathrm{t}}\right)_{i+1}=\mathbf{f}_{-} \mathbf{f}_{\mathrm{N}}^{-1}\left(\delta \mathbf{u}_{\mathrm{t}}\right)_{i} .
$$

The norm,

$$
\begin{aligned}
\left\|\left(\delta \mathbf{u}_{\mathrm{t}}\right)_{i+1}\right\| & =\left\|\mathbf{f}_{-} \mathbf{f}_{\mathrm{N}}^{-1}\left(\delta \mathbf{u}_{\mathrm{t}}\right)_{i}\right\| \\
& \leq\left\|\mathbf{f}_{-} \mathbf{f}_{\mathrm{N}}^{-1}\right\|\left\|\left(\delta \mathbf{u}_{\mathrm{t}}\right)_{i}\right\|,
\end{aligned}
$$

diminishes as long as

$$
\begin{aligned}
\omega_{\mathrm{fu}} & =\left\|\mathbf{f}_{-} \mathbf{f}_{\mathrm{N}}^{-1}\right\| \\
& =\max \sqrt{\lambda\left[\left(\mathbf{f}_{-} \mathbf{f}_{\mathrm{N}}^{-1}\right)^{\mathrm{t}}\left(\mathbf{f}_{-} \mathbf{f}_{\mathrm{N}}^{-1}\right)\right]}<1 .
\end{aligned}
$$

\subsection{The local level}

The partitioned element flexibility matrix in eqn (10) reflects the diagonal and off-diagonal constituents of the material flexibility matrix

$$
\phi_{\mathrm{N}} \Leftarrow \phi_{\mathrm{D}}+\phi_{-} .
$$

This transfers the relationship between natural stress and strain from the continuum to the spring cell

$$
\varepsilon_{\mathrm{t}}=\phi_{\mathrm{N}} \sigma_{\mathrm{c}} \Leftarrow \phi_{\mathrm{D}} \sigma_{\mathrm{c}}+\boldsymbol{\eta}_{\phi},
$$

where the elastic response of the cell members has been supplemented by the strain

$$
\boldsymbol{\eta}_{\phi}=\phi_{-} \boldsymbol{\sigma}_{\mathrm{c}}
$$

to complete the substitution of the continuum.

Equation (42) computes the strain for given stress. At prescribed strain the stress is approached iteratively

$$
\boldsymbol{\sigma}_{\mathrm{c}, i+1}=\phi_{\mathrm{D}}^{-1}\left(\varepsilon_{\mathrm{t}}-\phi_{-} \boldsymbol{\sigma}_{\mathrm{c}, i}\right) .
$$

Changes between consecutive cycles

$$
\left(\delta \boldsymbol{\sigma}_{\mathrm{c}}\right)_{i+1}=-\boldsymbol{\phi}_{\mathrm{D}}^{-1} \boldsymbol{\phi}_{-}\left(\delta \boldsymbol{\sigma}_{\mathrm{c}}\right)_{i},
$$

should diminish if

$$
\begin{aligned}
\omega_{\phi \sigma} & =\left\|\lambda\left(\phi_{\mathrm{D}}^{-1} \boldsymbol{\phi}_{-}\right)\right\| \\
& =\max \sqrt{\lambda\left[\left(\boldsymbol{\phi}_{\mathrm{D}}^{-1} \boldsymbol{\phi}_{-}\right)^{\mathrm{t}}\left(\boldsymbol{\phi}_{\mathrm{D}}^{-1} \boldsymbol{\phi}_{-}\right)\right]}<1 .
\end{aligned}
$$




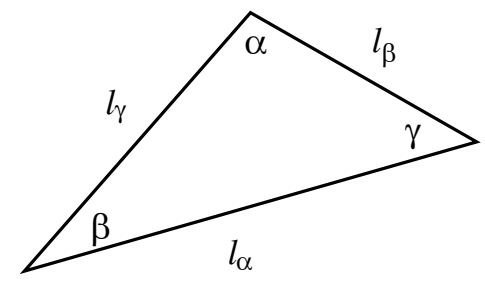

Figure 2: Geometry of triangular element.

A secondary scheme derives by expressing the supplement $\boldsymbol{\eta}_{\phi}$, eqn (43), in terms of the strain

$$
\boldsymbol{\eta}_{\phi}=\phi_{-} \phi_{\mathrm{N}}^{-1} \varepsilon_{\mathrm{t}} .
$$

Then at given strain the stress is determined directly in the cell context, while solution for the strain when the stress is imposed implies iteration

$$
\varepsilon_{\mathrm{t}, i+1}=\phi_{\mathrm{D}} \boldsymbol{\sigma}_{\mathrm{c}}+\phi_{-} \phi_{\mathrm{N}}^{-1} \varepsilon_{\mathrm{t}, i} .
$$

The norm of the difference

$$
\left(\delta \varepsilon_{\mathrm{t}}\right)_{i+1}=\phi_{-} \phi_{\mathrm{N}}^{-1}\left(\delta \varepsilon_{\mathrm{t}}\right)_{i},
$$

diminishes for

$$
\begin{aligned}
\omega_{\phi \varepsilon} & =\left\|\lambda\left(\phi_{-} \phi_{\mathrm{N}}^{-1}\right)\right\| \\
& =\max \sqrt{\lambda\left[\left(\phi_{-} \boldsymbol{\phi}_{\mathrm{N}}^{-1}\right)^{\mathrm{t}}\left(\phi_{-} \boldsymbol{\phi}_{\mathrm{N}}^{-1}\right)\right]}<1 .
\end{aligned}
$$

\section{The triangular element}

The general formalism is detailed for triangular configurations. Essentials of the description in [2] are recalled for completeness.

\subsection{Stress and strain}

The flexibility and the stiffness matrices of the elastic material are needed. The natural flexibility matrix pertaining to the triangle, Fig 2, reads,

$$
\begin{aligned}
& \phi_{\mathrm{N}}= \\
& \frac{1}{E}\left[\begin{array}{ccc}
1 & \cos ^{2} \gamma-\nu \sin ^{2} \gamma & \cos ^{2} \beta-\nu \sin ^{2} \beta \\
& 1 & \cos ^{2} \alpha-\nu \sin ^{2} \alpha \\
\text { sym } & & 1
\end{array}\right] \\
& =\frac{1}{E}\left[\begin{array}{ccc}
1 & c & b \\
& 1 & a \\
\operatorname{sym} & & 1
\end{array}\right] \text {. }
\end{aligned}
$$

The abbreviations

$$
\begin{aligned}
& c=\left(\cos ^{2} \gamma-\nu \sin ^{2} \gamma\right), \\
& b=\left(\cos ^{2} \beta-\nu \sin ^{2} \beta\right), \\
& a=\left(\cos ^{2} \alpha-\nu \sin ^{2} \alpha\right),
\end{aligned}
$$

help shorten subsequent expressions. Inversion computes the material stiffness matrix

$$
\begin{gathered}
\boldsymbol{\kappa}_{\mathrm{N}}=\phi_{\mathrm{N}}^{-1}= \\
=\frac{E}{\operatorname{Det}}\left[\begin{array}{ccc}
1-a^{2} & a b-c & a c-b \\
& 1-b^{2} & b c-a \\
\operatorname{sym} & & 1-c^{2}
\end{array}\right],
\end{gathered}
$$

with the determinant

$$
\text { Det }=\left|E \boldsymbol{\phi}_{\mathrm{N}}\right|=1-\left(a^{2}+b^{2}+c^{2}\right)+2 a b c .
$$

The operators of the iteration schemes are set up next. In the flexibility method the stress loop, eqn (44), is operated with the matrix

$$
\begin{aligned}
& \phi_{\mathrm{D}}^{-1} \phi_{-}= \\
& \phi_{\mathrm{D}}^{-1} \boldsymbol{\phi}_{\mathrm{N}}-\mathbf{I}=\left[\begin{array}{ccc}
0 & c & b \\
& 0 & a \\
\operatorname{sym} & & 0
\end{array}\right] \text {, }
\end{aligned}
$$

where $\mathbf{I}$ stands for the unity matrix and $\phi_{\mathrm{D}}^{-1}=E \mathbf{I}$. For the strain loop, eqn (48),

$$
\begin{gathered}
\boldsymbol{\phi}_{-} \boldsymbol{\phi}_{\mathrm{N}}^{-1}=\mathbf{I}-\boldsymbol{\phi}_{\mathrm{D}} \boldsymbol{\phi}_{\mathrm{N}}^{-1}=\frac{-1}{\operatorname{Det}} \times \\
{\left[\begin{array}{ccc}
b^{2}+c^{2}-2 a b c & a b-c & a c-b \\
\operatorname{sym} & c^{2}+a^{2}-2 a b c & b c-a \\
& & a^{2}+b^{2}-2 a b c
\end{array}\right] .}
\end{gathered}
$$

With this the convergence indicators $\omega_{\phi \sigma}$ for the stress loop, eqn (46), and $\omega_{\phi \varepsilon}$ for the strain loop, eqn (50), are computed in dependence of the shape of the triangle. Isolines are displayed in Fig 3; the region of convergence extends from the regular triangle and is limited by the unity line.

In the stiffness method, the strain loop, eqn (25), is operated with the matrix

$$
\begin{aligned}
\boldsymbol{\kappa}_{\mathrm{D}}^{-1} \boldsymbol{\kappa}_{-} & =\boldsymbol{\kappa}_{\mathrm{D}}^{-1} \boldsymbol{\kappa}_{\mathrm{N}}-\mathbf{I} \\
& =\left[\begin{array}{ccc}
0 & \frac{a b-c}{1-a^{2}} & \frac{a c-b}{1-a^{2}} \\
\frac{a b-c}{1-b^{2}} & 0 & \frac{b c-a}{1-b^{2}} \\
\frac{a c-b}{1-c^{2}} & \frac{b c-a}{1-c^{2}} & 0
\end{array}\right] .
\end{aligned}
$$

The stress in the loop of eqn (29) is transferred by the matrix

$$
\begin{gathered}
\boldsymbol{\kappa}_{-} \boldsymbol{\kappa}_{\mathrm{N}}^{-1}=\mathbf{I}-\boldsymbol{\kappa}_{\mathrm{D}} \boldsymbol{\kappa}_{\mathrm{N}}^{-\mathbf{1}}=\frac{-\mathbf{1}}{\mathrm{Det}} \times \\
{\left[\begin{array}{ccc}
b^{2}+c^{2}-2 a b c & \left(1-a^{2}\right) c & \left(1-a^{2}\right) b \\
\left(1-b^{2}\right) c & c^{2}+a^{2}-2 a b c & \left(1-b^{2}\right) a \\
\left(1-c^{2}\right) b & \left(1-c^{2}\right) a & a^{2}+b^{2}-2 a b c
\end{array}\right] .}
\end{gathered}
$$




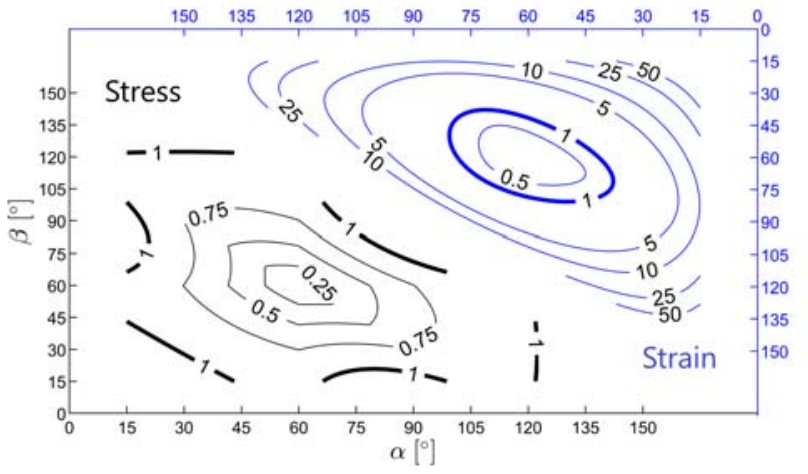

Figure 3: Isolines of the convergence indicators for varying triangular shape in the material flexibility context. Left: Iteration of stress $\left(\omega_{\phi \sigma}\right)$. Right: Iteration of strain $\left(\omega_{\phi \varepsilon}\right)$.

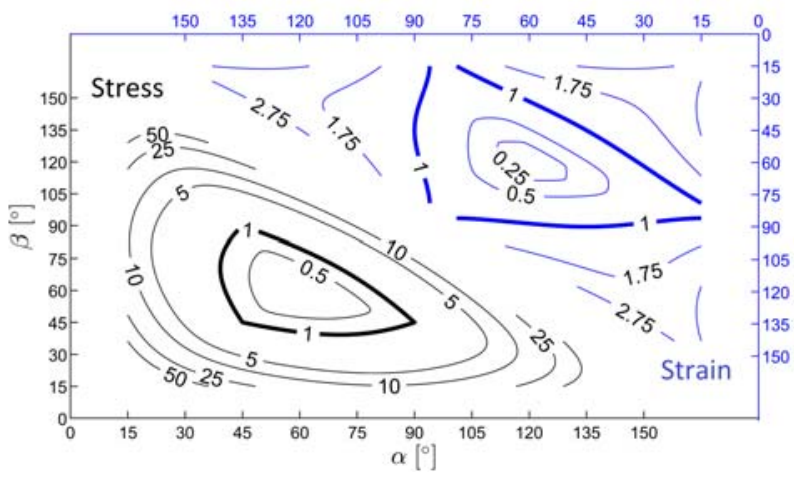

Figure 4: Isolines of the onvergence indicators for varying triangular shape in the material stiffness context. Left: Iteration of stress $\left(\omega_{\kappa \sigma}\right)$. Right: Iteration of strain $\left(\omega_{\kappa \varepsilon}\right)$.

The non-symmetric matrices enter the determination of the convergence indicators $\omega_{\kappa \varepsilon}$ for the strain loop, eqn (27), and $\omega_{\kappa \sigma}$ for the stress loop, eqn (31). Figure 4 displays isolines confining the region of convergence in dependence of the shape of the triangle.

It may be observed that the modulus of elasticity does not enter none of the iteration operators. It does not affect the convergence behaviour in contrast to the coefficient of lateral contraction. The latter is set to $\nu=1 / 3$ in all numerical evaluations.

\subsection{Displacement and force}

The stiffness- and flexibility matrices of the elastic material enter the operators of the finite element iteration.

The flexibility method: The solution for the force $\mathbf{S}_{\mathrm{c}}$ at fixed displacement $\mathbf{u}_{\mathrm{t}}$ follows the scheme of eqn (32). On consideration of eqn (10) for the finite element the pertaining operator is

$$
\begin{array}{r}
\mathbf{f}_{\mathrm{D}}^{-1} \mathbf{f}_{-}=\left(V \mathbf{l}^{-1} \boldsymbol{\phi}_{\mathrm{D}}^{-1} \mathbf{l}^{-1}\right)\left(V^{-1} \mathbf{l} \boldsymbol{\phi}_{-} \mathbf{l}\right) \\
=\mathbf{l}^{-1} \boldsymbol{\phi}_{\mathrm{D}}^{-1} \boldsymbol{\phi}_{-} \mathbf{l} .
\end{array}
$$

The core of the expression is given for the triangle by eqn (55) and with the trigonometric relation for the lengths

$$
\mathbf{l}=\left[\begin{array}{l}
l_{\alpha} \\
l_{\beta} \\
l_{\gamma}
\end{array}\right]=2 R\left[\begin{array}{c}
\sin \alpha \\
\sin \beta \\
\sin \gamma
\end{array}\right]
$$

the operator is obtained as

$$
\mathbf{f}_{\mathrm{D}}^{-1} \mathbf{f}_{-}=\left[\begin{array}{ccc}
0 & c \frac{\sin \beta}{\sin \alpha} & b \frac{\sin \gamma}{\sin \alpha} \\
c \frac{\sin \alpha}{\sin \beta} & 0 & a \frac{\sin \gamma}{\sin \beta} \\
b \frac{\sin \alpha}{\sin \gamma} & a \frac{\sin \beta}{\sin \gamma} & 0
\end{array}\right]
$$

It is noted that eventually neither the element volume $V$ nor the circumscribing radius $R$ appear. The iteration operator depends solely on the shape of the triangle and on the coefficient of lateral contraction. This proves true for what follows as well.

The solution for the displacement $\mathbf{u}_{\mathrm{t}}$ at fixed force $\mathbf{S}_{\mathrm{c}}$ follows the scheme of eqn (37). The progress of the iteration is governed by the matrix

$$
\begin{array}{r}
\mathbf{f}_{-} \mathbf{f}_{\mathrm{N}}^{-1}=\left(V^{-1} \mathbf{l} \phi_{-} \mathbf{l}\right)\left(V \mathbf{l}^{-1} \boldsymbol{\phi}_{\mathrm{N}}^{-1} \mathbf{l}^{-1}\right) \\
=\mathbf{l} \phi_{-} \boldsymbol{\phi}_{\mathrm{N}}^{-1} \mathbf{l}^{-1} .
\end{array}
$$

With the core from eqn (56) the operator becomes

$$
\begin{gathered}
\mathbf{f}_{-} \mathbf{f}_{\mathrm{N}}^{-\mathbf{1}}=\frac{\mathbf{- 1}}{\operatorname{Det}} \times \\
{\left[\begin{array}{ccc}
b^{2}+c^{2}-2 a b c & (a b-c) \frac{\sin \alpha}{\sin \beta} & (a c-b) \frac{\sin \alpha}{\sin \gamma} \\
(a b-c) \frac{\sin \beta}{\sin \alpha} & c^{2}+a^{2}-2 a b c & (b c-a) \frac{\sin \beta}{\sin \gamma} \\
(a c-b) \frac{\sin \gamma}{\sin \alpha} & (b c-a) \frac{\sin \gamma}{\sin \beta} & a^{2}+b^{2}-2 a b c
\end{array}\right] .}
\end{gathered}
$$

The matrices in eqn (61) and eqn (63) determine the convergence indicators $\omega_{\mathrm{fS}}$ for the force loop, eqn (35), and $\omega_{\mathrm{fu}}$ for the displacement loop, eqn (40). Isolines are displayed in Fig 5.

The stiffness method: The displacement $\mathbf{u}_{\mathrm{t}}$ at fixed force $\mathbf{S}_{\mathrm{c}}$ is approached by the recursive scheme of eqn (12). With reference to eqn (6) for the element stiffness the iteration matrix is

$$
\begin{array}{r}
\mathbf{k}_{\mathrm{D}}^{-1} \mathbf{k}_{-}=\left(V^{-1} \mathbf{l} \boldsymbol{\kappa}_{\mathrm{D}}^{-1} \mathbf{l}\right)\left(V \mathbf{l}^{-1} \boldsymbol{\kappa}_{-} \mathbf{l}^{-1}\right) \\
=\mathbf{l} \boldsymbol{\kappa}_{\mathrm{D}}^{-1} \boldsymbol{\kappa}_{-} \mathbf{l}^{-1}
\end{array}
$$




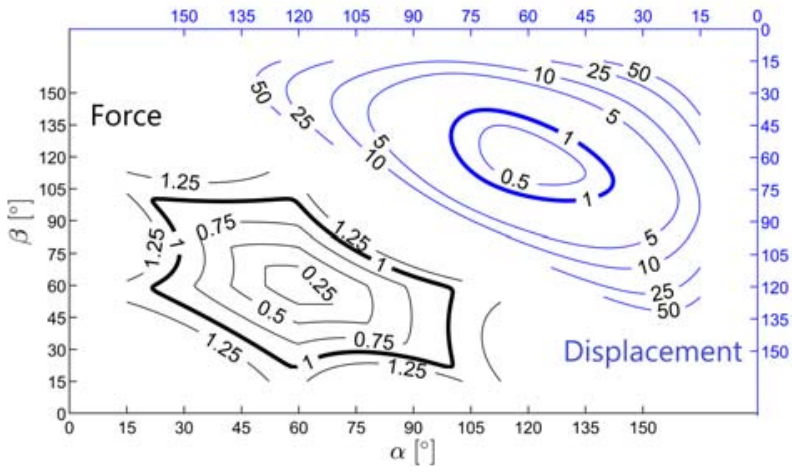

Figure 5: Isolines of the convergence indicators for varying triangular shape in the element flexibility context. Left: Iteration of force $\left(\omega_{\mathrm{fS}}\right)$. Right: Iteration of displacement $\left(\omega_{\mathrm{fu}}\right)$.

The core of the expression is given for the triangle in eqn (57), and with eqn (60) for the side lengths,

$$
\mathbf{k}_{\mathrm{D}}^{-1} \mathbf{k}_{-}=\left[\begin{array}{ccc}
0 & \frac{a b-c}{1-a^{2}} \frac{\sin \alpha}{\sin \beta} & \frac{a c-b}{1-a^{2}} \frac{\sin \alpha}{\sin \gamma} \\
\frac{a b-c}{1-b^{2}} \sin \beta & 0 & \frac{b c-a}{1-b^{2}} \frac{\sin \beta}{\sin \gamma} \\
\frac{a c-b}{1-c^{2}} \frac{\sin \gamma}{\sin \alpha} & \frac{b c-a}{1-c^{2}} \frac{\sin \gamma}{\sin \beta} & 0
\end{array}\right]
$$

The force $\mathbf{S}_{\mathrm{c}}$ at constant displacement $\mathbf{u}_{\mathrm{t}}$, eqn (18), is iterated with the matrix

$$
\begin{array}{r}
\mathbf{k}_{-} \mathbf{k}_{\mathrm{N}}^{-1}=\left(V \mathbf{l}^{-1} \boldsymbol{\kappa}_{-} \mathbf{l}^{-1}\right)\left(V^{-1} \mathbf{l} \boldsymbol{\kappa}_{\mathrm{N}}^{-1} \mathbf{l}\right) \\
=\mathbf{l}^{-1} \boldsymbol{\kappa}_{-} \boldsymbol{\kappa}_{\mathrm{N}}^{-1} \mathbf{l}
\end{array}
$$

With eqn (58) the operator matrix becomes

$$
\begin{gathered}
\mathbf{k}_{-} \mathbf{k}_{\mathrm{N}}^{-1}=\frac{-1}{\operatorname{Det}} \times \\
{\left[\begin{array}{ccc}
b^{2}+c^{2}-2 a b c & \left(1-a^{2}\right) c \frac{\sin \beta}{\sin \alpha} & \left(1-a^{2}\right) b \frac{\sin \gamma}{\sin \alpha} \\
\left(1-b^{2}\right) c \frac{\sin \alpha}{\sin \beta} & c^{2}+a^{2}-2 a b c & \left(1-b^{2}\right) a \frac{\sin \gamma}{\sin \beta} \\
\left(1-c^{2}\right) b \frac{\sin \alpha}{\sin \gamma} & \left(1-c^{2}\right) a \frac{\sin \beta}{\sin \gamma} & a^{2}+b^{2}-2 a b c
\end{array}\right] .}
\end{gathered}
$$

The convergence indicator $\omega_{\mathrm{ku}}$ for the displacement loop is determined by eqn (16), and $\omega_{\mathrm{kS}}$ for the force loop by eqn (21). Figure 6 displays isolines.

\subsection{Discussion}

The number of iterations for completing substitution depends on the shape of the element. It increases from zero at the ideal configuration of the regular triangle up to divergent solutions beyond the convergence limit. The latter depends on the cell concept and on the problem to solve.

Inspection of the displayed distribution of the convergence indicators shows a larger region for the primary operators emanating directly from the partitioning of the material constitutive matrices. In the

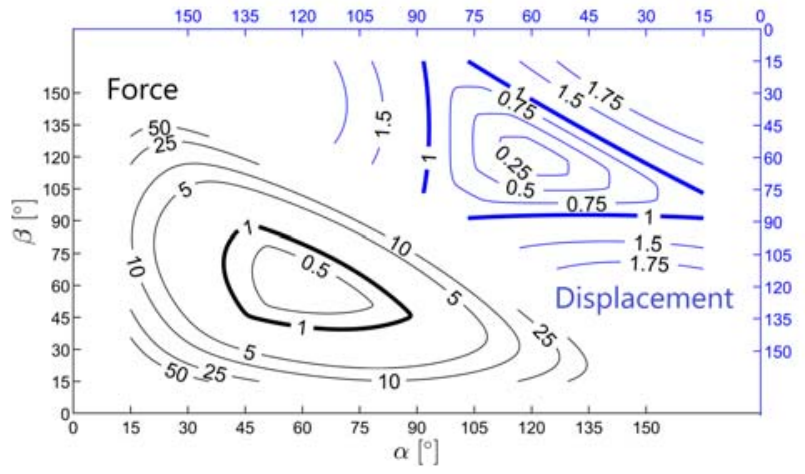

Figure 6: Isolines of the convergence indicators for varying triangular shape in the element stiffness context. Left: Iteration of force $\left(\omega_{\mathrm{kS}}\right)$. Right: Iteration of displacement $\left(\omega_{\mathrm{ku}}\right)$.

flexibility context, Fig 3, this applies to the iteration of the stress, eqn (44), as contrasted to the iteration of the strain, eqn (48). Analogously in the stiffness context, Fig 4, the strain iteration, eqn (25), covers tiangular shapes to a larger extent than the iteration of the stress, eqn (29). Comparison of Fig 4 and Fig 3 indicates a superiority of the flexibility concept against the stiffness concept when comparing different conditions: straining in the former with stressing in the latter. The secondary, deduced variants of the two concepts are close to each other.

It is worth relating the convergence indicators with the eigenvalues of the loop operators on the grounds of matrix theory [11]. The eigenvalues of the flexibility matrix under exclusion of the elastic modulus are obtained from the characteristic equation

$$
\begin{gathered}
\left|E \boldsymbol{\phi}_{\mathrm{N}}-\lambda \mathbf{I}\right|= \\
(1-\lambda)^{3}-\left(a^{2}+b^{2}+c^{2}\right)(1-\lambda)+2 a b c=0 .
\end{gathered}
$$

With the solution $\lambda=\lambda\left(E \phi_{\mathrm{N}}\right)$ the eigenvalues of other participating matrices can be deduced. For the stiffness matrix by inversion,

$$
\lambda\left(\frac{1}{E} \kappa_{\mathrm{N}}\right)=\lambda\left[\left(E \boldsymbol{\phi}_{\mathrm{N}}\right)^{-1}\right]=\frac{1}{\lambda\left(E \boldsymbol{\phi}_{\mathrm{N}}\right)} .
$$

Attention now is paid to the iteration schemes. In the flexibility method the operator matrix of the stress loop, eqn (55), is symmetric with eigenvalues

$$
\lambda\left(\phi_{\mathrm{D}}^{-1} \boldsymbol{\phi}_{-}\right)=\lambda\left(E \boldsymbol{\phi}_{\mathrm{N}}\right)-1 .
$$

For the strain loop, eqn (56),

$$
\lambda\left(\phi_{-} \phi_{\mathrm{N}}^{-1}\right)=1-\frac{1}{\lambda\left(E \phi_{\mathrm{N}}\right)} .
$$


Equations (71) and (70) have in common the eigenvalue $\lambda\left(E \phi_{\mathrm{N}}\right)$. Eliminating,

$$
\lambda\left(\phi_{-} \boldsymbol{\phi}_{\mathrm{N}}^{-1}\right)=\frac{\lambda\left(\boldsymbol{\phi}_{\mathrm{D}}^{-1} \boldsymbol{\phi}_{-}\right)}{1+\lambda\left(\boldsymbol{\phi}_{\mathrm{D}}^{-1} \boldsymbol{\phi}_{-}\right)} .
$$

For the symmetric operators of the flexibility procedures the spectral norm equals the spectral radius, such that the convergence indicators by eqn (46) and eqn (50) are identified as

$$
\omega_{\phi \sigma}=\max \left|\lambda\left(\phi_{\mathrm{D}}^{-1} \phi_{-}\right)\right|=\max \left|\lambda\left(E \phi_{\mathrm{N}}\right)-1\right|,
$$

and

$$
\omega_{\phi \varepsilon}=\max \left|\lambda\left(\phi_{-} \phi_{\mathrm{N}}^{-1}\right)\right|=\max \left|1-\frac{1}{\lambda\left(E \phi_{\mathrm{N}}\right)}\right| .
$$

The stiffness procedures are operated with the nonsymmetric matrices of eqn (57) and eqn (58). In this case the spectral norms, the convergence indicators of eqn (27) and eqn (31), satisfy the inequalities

$$
\omega_{\kappa \varepsilon}>\max \left|\lambda\left(\boldsymbol{\kappa}_{\mathrm{D}}^{-1} \boldsymbol{\kappa}_{-}\right)\right| .
$$

and

$$
\omega_{\kappa \sigma}>\max \left|\lambda\left(\boldsymbol{\kappa}_{-} \boldsymbol{\kappa}_{\mathrm{N}}^{-1}\right)\right|,
$$

The transition to the finite element does not have a dramatic effect. The eigenvalues of the finite element matrices and of the material matrices are equal. With reference to eqns (59) and (62) in the flexibility context

$$
\begin{aligned}
& \lambda\left(\mathbf{f}_{\mathrm{D}}^{-1} \mathbf{f}_{-}\right)=\lambda\left(\mathbf{l}^{-1} \boldsymbol{\phi}_{\mathrm{D}}^{-1} \boldsymbol{\phi}_{-} \mathbf{l}\right)=\lambda\left(\boldsymbol{\phi}_{\mathrm{D}}^{-1} \boldsymbol{\phi}_{-}\right), \\
& \lambda\left(\mathbf{f}_{-} \mathbf{f}_{\mathrm{N}}^{-\mathbf{1}}\right)=\lambda\left(\mathbf{l} \boldsymbol{\phi}_{-} \boldsymbol{\phi}_{\mathrm{N}}^{-\mathbf{1}} \mathbf{l}^{-\mathbf{1}}\right)=\lambda\left(\boldsymbol{\phi}_{-} \boldsymbol{\phi}_{\mathrm{N}}^{-\mathbf{1}}\right) .
\end{aligned}
$$

From eqns (64) and (66) for the stiffness operators

$$
\begin{gathered}
\lambda\left(\mathbf{k}_{\mathrm{D}}^{-1} \mathbf{k}_{-}\right)=\lambda\left(\mathbf{l} \boldsymbol{\kappa}_{\mathrm{D}}^{-1} \boldsymbol{\kappa}_{-} \mathbf{l}^{-1}\right)=\lambda\left(\boldsymbol{\kappa}_{\mathrm{D}}^{-1} \boldsymbol{\kappa}_{-}\right), \\
\lambda\left(\mathbf{k}_{-} \mathbf{k}_{\mathrm{N}}^{-1}\right)=\lambda\left(\mathbf{l}^{-1} \boldsymbol{\kappa}_{-} \boldsymbol{\kappa}_{\mathrm{N}}^{-1} \mathbf{l}\right)=\lambda\left(\boldsymbol{\kappa}_{-} \boldsymbol{\kappa}_{\mathrm{N}}^{-1}\right) .
\end{gathered}
$$

Since the flexibility operators for the element are nonsymmetric, from eqns (35) and (40),

$$
\begin{gathered}
\omega_{\mathrm{fS}}>\max \left|\lambda\left(\mathbf{f}_{\mathrm{D}}^{-1} \mathbf{f}_{-}\right)\right|=\max \left|\lambda\left(\boldsymbol{\phi}_{\mathrm{D}}^{-1} \boldsymbol{\phi}_{-}\right)\right|, \\
\omega_{\mathrm{fu}}>\max \left|\lambda\left(\mathbf{f}_{-} \mathbf{f}_{\mathrm{N}}^{-1}\right)\right|=\max \left|\lambda\left(\boldsymbol{\phi}_{-} \boldsymbol{\phi}_{\mathrm{N}}{ }^{-1}\right)\right| .
\end{gathered}
$$

Comparison with eqns (73) and (74) predicts a reduced region of convergence for the element:

$$
\begin{gathered}
\omega_{\mathrm{fS}}>\omega_{\phi \sigma}, \\
\omega_{\mathrm{fu}}>\omega_{\phi \varepsilon} .
\end{gathered}
$$

For the convergence indicators of the stiffness procedure, eqns (16) and (21), one obtains

$$
\begin{gathered}
\omega_{\mathrm{ku}}>\max \left|\lambda\left(\mathbf{k}_{\mathrm{D}}^{-1} \mathbf{k}_{-}\right)\right|=\max \left|\lambda\left(\boldsymbol{\kappa}_{\mathrm{D}}^{-1} \boldsymbol{\kappa}_{-}\right)\right|, \\
\lambda_{\mathrm{kS}}>\max \left|\lambda\left(\mathbf{k}_{-} \mathbf{k}_{\mathrm{N}}^{-1}\right)\right|=\max \left|\lambda\left(\boldsymbol{\kappa}_{-} \boldsymbol{\kappa}_{\mathrm{N}}^{-1}\right)\right|,
\end{gathered}
$$

which compares with eqns (75) and (76).

As seen from Fig 5 and Fig 6 the convergence region for the primarily partitioned element operators shrinks with respect to the material level, Fig 3 and Fig 4. That for the secondary, deduced schemes remains almost unaffected; the overall tendency similar. The above discussion on the plane triangle transfers equally to the tetrahedral element.

\section{Numerical demonstration}

\subsection{Preliminaries}

\subsubsection{Geometry and loading}

The theory is implemented for a triangle with

$$
\alpha=90^{\circ}, \beta=60^{\circ}, \gamma=30^{\circ} .
$$

This configuration comprises convergent solutions near the limit and shows the divergence experienced beyond it.

Evaluation of eqn (51) and eqn (53) for $\nu=1 / 3$, $E=1$ specifies the flexibility matrix and the stiffness matrix of the elastic material:

$$
\phi_{\mathrm{N}}=\left[\begin{array}{ccc}
1 & \frac{2}{3} & 0 \\
& 1 & -\frac{1}{3} \\
\text { sym } & & 1
\end{array}\right]
$$

with eigenvalues

$$
\lambda\left(\phi_{\mathrm{N}}\right)=\left\{\begin{array}{c}
1+\frac{\sqrt{5}}{3}=1.745 \\
1 \\
1-\frac{\sqrt{5}}{3}=0.255
\end{array},\right.
$$

and

$$
\boldsymbol{\kappa}_{\mathrm{N}}=\left[\begin{array}{ccc}
2 & -\frac{3}{2} & -\frac{1}{2} \\
& \frac{9}{4} & \frac{3}{4} \\
\operatorname{sym} & & \frac{5}{4}
\end{array}\right]
$$

with the eigenvalues obtained by inversion

$$
\lambda\left(\boldsymbol{\kappa}_{\mathrm{N}}\right)=\lambda^{-1}\left(\boldsymbol{\phi}_{\mathrm{N}}\right)=\left\{\begin{array}{c}
\frac{3}{3-\sqrt{5}}=3.927 \\
1 \\
\frac{3}{3+\sqrt{5}}=0.573
\end{array} .\right.
$$

The numerical procedure is carried out for a state of shear stress $\sigma_{12}=\sqrt{3}$ defined in the Cartesian 
reference system $\mathrm{O}-1,2$ with axes along the sides of the triangle opposed to the angles $\beta$ and $\gamma$. Standard transformation relates the stress components to the natural ones

$$
\left[\begin{array}{l}
\sigma_{11} \\
\sigma_{22} \\
\sigma_{12}
\end{array}\right]=\left[\begin{array}{ccc}
\frac{3}{4} & 1 & 0 \\
\frac{1}{4} & 0 & 1 \\
\frac{\sqrt{3}}{4} & 0 & 0
\end{array}\right]\left[\begin{array}{c}
\sigma_{\mathrm{c}}^{\alpha} \\
\sigma_{\mathrm{c}}^{\beta} \\
\sigma_{\mathrm{c}}^{\gamma}
\end{array}\right]=\left[\begin{array}{c}
0 \\
0 \\
\sqrt{3}
\end{array}\right] .
$$

The entities in the transformation matrix stand for $\cos (\vartheta, i) \cos (\vartheta, j), \vartheta=\alpha, \beta, \gamma ; i, j=1,2$ according to the indexing of the vector arrays. Inversion specifies the natural equivalent of the Cartesian stress:

$$
\left\{\sigma_{\mathrm{c}}^{\alpha} \sigma_{\mathrm{c}}^{\beta} \sigma_{\mathrm{c}}^{\gamma}\right\}=\{4-3-1\} .
$$

The associated natural strain is computed with the material flexibility matrix of eqn (83)

$$
\left\{\varepsilon_{\mathrm{t}}^{\alpha} \varepsilon_{\mathrm{t}}^{\beta} \varepsilon_{\mathrm{t}}^{\gamma}\right\}=\left\{\begin{array}{lll}
2 & 0 & 0
\end{array}\right\} .
$$

Since geometrical dimensions do not affect the convergence behaviour, the step to the finite element is done with the unity values $V=1,2 R=1$, and the side lengths from eqn (60) as in the diagonal matrix

$$
\mathbf{l}=\left\lceil 1 \frac{\sqrt{3}}{2} \frac{1}{2}\right\rfloor \text {. }
$$

The forces $\mathbf{S}_{\mathrm{c}}$ along the sides result from the stress in eqn (88)

$$
\mathbf{S}_{\mathrm{c}}=V \mathbf{1}^{-1} \boldsymbol{\sigma}_{\mathrm{c}}=\left[\begin{array}{c}
S_{\mathrm{c}}^{\alpha} \\
S_{\mathrm{c}}^{\beta} \\
S_{\mathrm{c}}^{\gamma}
\end{array}\right]=\left[\begin{array}{c}
4 \\
-2 \sqrt{3} \\
-2
\end{array}\right],
$$

and the elongations $\mathbf{u}_{\mathrm{t}}$ are associated with the strain in eqn (89)

$$
\mathbf{u}_{\mathrm{t}}=\mathbf{l} \boldsymbol{\varepsilon}_{\mathrm{t}}=\left[\begin{array}{c}
u_{\mathrm{t}}^{\alpha} \\
u_{\mathrm{t}}^{\beta} \\
u_{\mathrm{t}}^{\gamma}
\end{array}\right]=\left[\begin{array}{l}
2 \\
0 \\
0
\end{array}\right] .
$$

The flexibility matrix and the stiffness matrix relating the above element quantities are

$$
\mathbf{f}_{\mathrm{N}}=\mathbf{l} \phi_{\mathrm{N}} \mathbf{l}=\left[\begin{array}{ccc}
1 & \frac{\sqrt{3}}{3} & 0 \\
& \frac{3}{4} & -\frac{\sqrt{3}}{12} \\
\operatorname{sym} & & \frac{1}{4}
\end{array}\right],
$$

and

$$
\mathbf{k}_{\mathrm{N}}=\mathbf{l}^{-1} \boldsymbol{\kappa}_{\mathrm{N}} \mathbf{l}^{-1}=\left[\begin{array}{ccc}
2 & -\sqrt{3} & -1 \\
& 3 & \sqrt{3} \\
\text { sym } & & 5
\end{array}\right],
$$

with eigenvalues

$$
\lambda\left(\mathbf{f}_{\mathrm{N}}\right)=1.472,0.377,0.150,
$$

and by inversion in reverse order,

$$
\lambda\left(\mathbf{k}_{\mathrm{N}}\right)=6.672,2.649,0.679 .
$$

\subsubsection{Cell substitutes}

Essentially, the two explicit spring cell substitutes of the finite element are based on the diagonal entities of the constitutive matrices of the elastic material: the flexibility matrix, eqn (83), and the stiffness matrix, eqn (85).

A measure regarding the coverage of the finite element has been introduced in [6] as the Rayleigh quotient defined by the elastic energy in the activated units after standardization with the element volume, the modulus of elasticity at fixed contraction coefficient and the magnitude of the action. Specifically, with reference to the straining mode of eqn (89), the quantities are:

For the finite element

$$
\left(\bar{w}_{\mathrm{N}}\right)_{\varepsilon}=\frac{\varepsilon_{\mathrm{t}}^{\mathrm{t}}\left(\frac{1}{E} \boldsymbol{\kappa}_{\mathrm{N}}\right) \varepsilon_{\mathrm{t}}}{\varepsilon_{\mathrm{t}}^{\mathrm{t}} \varepsilon_{\mathrm{t}}}=2,
$$

for the flexibility cell

$$
\left(\bar{w}_{\mathrm{f}}\right)_{\varepsilon}=\frac{\varepsilon_{\mathrm{t}}^{\mathrm{t}}\left(E \phi_{\mathrm{D}}\right)^{-1} \varepsilon_{\mathrm{t}}}{\varepsilon_{\mathrm{t}}^{\mathrm{t}} \varepsilon_{\mathrm{t}}}=1
$$

and for the stiffness cell

$$
\left(\bar{w}_{\mathrm{k}}\right)_{\varepsilon}=\frac{\varepsilon_{\mathrm{t}}^{\mathrm{t}}\left(\frac{1}{E} \kappa_{\mathrm{D}}\right) \varepsilon_{\mathrm{t}}^{\mathrm{t}}}{\varepsilon_{\mathrm{t}}^{\mathrm{t}} \varepsilon}=2 .
$$

The values of the Rayleigh quotient lie within the spectra of the involved matrices, and in the present constellation of geometry and straining are seen to favour the stiffness cell.

The stressing mode of eqn (88) gives for the finite element

$$
\left(\bar{w}_{\mathrm{N}}\right)_{\sigma}=\frac{\boldsymbol{\sigma}_{\mathrm{c}}^{\mathrm{t}}\left(E \boldsymbol{\phi}_{\mathrm{N}}\right) \boldsymbol{\sigma}_{\mathrm{c}}}{\boldsymbol{\sigma}_{\mathrm{c}}^{\mathrm{t}} \boldsymbol{\sigma}_{\mathrm{c}}}=0.308,
$$

for the flexibility cell

$$
\left(\bar{w}_{\mathrm{f}}\right)_{\sigma}=\frac{\boldsymbol{\sigma}_{\mathrm{c}}^{\mathrm{t}}\left(E \boldsymbol{\phi}_{\mathrm{D}}\right) \boldsymbol{\sigma}_{\mathrm{c}}}{\boldsymbol{\sigma}_{\mathrm{c}}^{\mathrm{t}} \boldsymbol{\sigma}_{\mathrm{c}}}=1
$$

and for the stiffness cell

$$
\left(\bar{w}_{\mathrm{k}}\right)_{\sigma}=\frac{\boldsymbol{\sigma}_{\mathrm{c}}^{\mathrm{t}}\left(\frac{1}{E} \boldsymbol{\kappa}_{\mathrm{D}}\right)^{-1} \boldsymbol{\sigma}_{\mathrm{c}}}{\boldsymbol{\sigma}_{\mathrm{c}}^{\mathrm{t}} \boldsymbol{\sigma}_{\mathrm{c}}}=0.492 .
$$

The values of the Rayleigh quotient computed in terms of the stress confirm an apparent superiority of the stiffness cell in reproducing the elastic energy of the element as concluded before for the straining. 


\subsection{The flexibility procedure}

\subsubsection{Stress and strain}

The scheme of eqn (44) for the stress at fixed strain is implemented with the actual data

$$
\left[\begin{array}{l}
\sigma_{\mathrm{c}}^{\alpha} \\
\sigma_{\mathrm{c}}^{\beta} \\
\sigma_{\mathrm{c}}^{\gamma}
\end{array}\right]_{i+1}=\left[\begin{array}{l}
2 \\
0 \\
0
\end{array}\right]-\left[\begin{array}{ccc}
0 & \frac{2}{3} & 0 \\
\frac{2}{3} & 0 & -\frac{1}{3} \\
0 & -\frac{1}{3} & 0
\end{array}\right]\left[\begin{array}{l}
\sigma_{\mathrm{c}}^{\alpha} \\
\sigma_{\mathrm{c}}^{\beta} \\
\sigma_{\mathrm{c}}^{\gamma}
\end{array}\right]_{i} .
$$

The eigenvalues of the symmetric operator matrix obtained with $\lambda\left(\phi_{\mathrm{N}}\right)$ from eqn (84)

$$
\lambda\left(\phi_{\mathrm{D}}^{-1} \phi_{-}\right)=\lambda\left(\phi_{\mathrm{N}}\right)-1=\left\{\begin{array}{c}
\frac{\sqrt{5}}{3}=0.745 \\
0 \\
-\frac{\sqrt{5}}{3}=-0.745
\end{array},\right.
$$

determine the spectral norm to $\omega_{\phi \sigma}=0.745<1$. The solution is expected to converge. Figure 7 shows the cycle deviation during the course of the iteration process as the quotient of Euclidean norms

$$
\Delta=\frac{\left\|\boldsymbol{\sigma}_{\mathrm{c}, i+1}-\boldsymbol{\sigma}_{\mathrm{c}, i}\right\|}{\left\|\boldsymbol{\sigma}_{\mathrm{c}, i}-\boldsymbol{\sigma}_{\mathrm{c}, i-1}\right\|}
$$

and the variation of the relative error

$$
\epsilon=\frac{\left\|\sigma_{\mathrm{c}}-\boldsymbol{\sigma}_{\mathrm{c}, i}\right\|}{\left\|\boldsymbol{\sigma}_{\mathrm{c}}\right\|}
$$

where $\boldsymbol{\sigma}_{\mathrm{c}}$ is the targeted stress of eqn (88). Contrasting with the iterative solution after 15 cycles

$$
\begin{aligned}
\boldsymbol{\sigma}_{\mathrm{c}, 15} & =\{3.967-2.951-0.984\} \\
\boldsymbol{\sigma}_{\mathrm{c}} & =\{4.000-3.000-1.000\} .
\end{aligned}
$$

The iteration scheme of eqn (48) for the strain at the imposed stress becomes

$$
\left[\begin{array}{c}
\varepsilon_{\mathrm{t}}^{\alpha} \\
\varepsilon_{\mathrm{t}}^{\beta} \\
\varepsilon_{\mathrm{t}}^{\gamma}
\end{array}\right]_{i+1}=\left[\begin{array}{c}
4 \\
-3 \\
-1
\end{array}\right]+\left[\begin{array}{ccc}
-1 & \frac{3}{2} & \frac{1}{2} \\
\frac{3}{2} & -\frac{5}{4} & -\frac{3}{4} \\
\frac{1}{2} & -\frac{3}{4} & -\frac{1}{4}
\end{array}\right]\left[\begin{array}{c}
\varepsilon_{\mathrm{t}}^{\alpha} \\
\varepsilon_{\mathrm{t}}^{\beta} \\
\varepsilon_{\mathrm{t}}^{\gamma}
\end{array}\right]_{i} .
$$

The operation matrix is symmetric with eigenvalues computed using those in eqn (84):

$$
\lambda\left(\phi_{-} \phi_{\mathrm{N}}^{-1}\right)=1-\frac{1}{\lambda\left(\phi_{\mathrm{N}}\right)}=\left\{\begin{array}{c}
\frac{\sqrt{5}}{3+\sqrt{5}}=0.427 \\
0 \\
\frac{-\sqrt{5}}{3-\sqrt{5}}=-2.927
\end{array} .\right.
$$

The spectral norm $\omega_{\phi \varepsilon}=2.927>1$, is indicative of divergence. Figure 8 demonstrates.

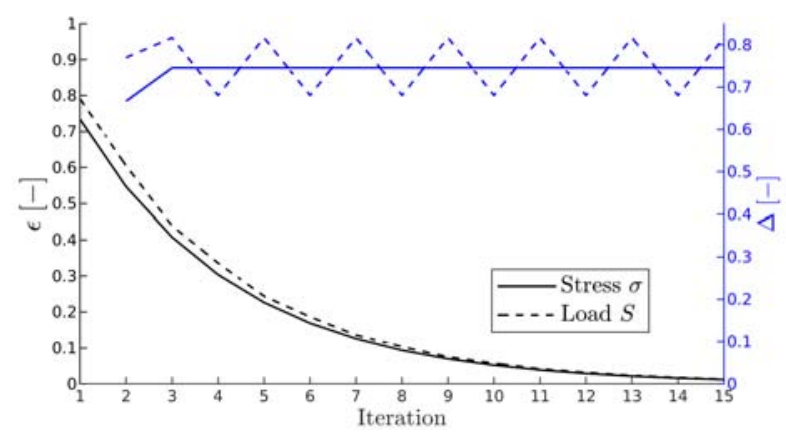

Figure 7: Stress and force in the iterative flexibility cell context. Difference quotient $\Delta$ at constant level, diminishing error $\epsilon$.

\subsubsection{Force and displacement}

The iterative scheme for the forces while the displacement is fixed, eqn (32), is implemented as

$$
\begin{aligned}
{\left[\begin{array}{l}
S_{\mathrm{c}}^{\alpha} \\
S_{\mathrm{c}}^{\beta} \\
S_{\mathrm{c}}^{\gamma}
\end{array}\right]_{i+1}=\left[\begin{array}{l}
2 \\
0 \\
0
\end{array}\right] } \\
-\left[\begin{array}{ccc}
0 & \frac{\sqrt{3}}{3} & 0 \\
\frac{4 \sqrt{3}}{9} & 0 & -\frac{\sqrt{3}}{9} \\
0 & -\frac{\sqrt{3}}{3} & 0
\end{array}\right]\left[\begin{array}{c}
S_{\mathrm{c}}^{\alpha} \\
S_{\mathrm{c}}^{\beta} \\
S_{\mathrm{c}}^{\gamma}
\end{array}\right]_{i} .
\end{aligned}
$$

The spectral norm for the operator matrix amounts to

$$
\omega_{\mathrm{fS}}=0.8165>0.745=\omega_{\phi \sigma},
$$

which confirms eqn (80) and is still $<1$ such that the solution should converge. The result after 15 cycles compares with the expected force:

$$
\begin{aligned}
\mathbf{S}_{\mathrm{c}, 15} & =\{3.967-3.408-1.967\} \\
\mathbf{S}_{\mathrm{c}} & =\{4.000-3.464-2.000\} .
\end{aligned}
$$

Iteration for the displacement at fixed force is by the scheme of eqn (37)

$$
\left[\begin{array}{l}
u_{\mathrm{t}}^{\alpha} \\
u_{\mathrm{t}}^{\beta} \\
u_{\mathrm{t}}^{\gamma}
\end{array}\right]_{i+1}=\left[\begin{array}{c}
4 \\
-\frac{3 \sqrt{3}}{2} \\
-\frac{1}{2}
\end{array}\right]
$$

The spectral norm of the operator matrix

$$
\omega_{\mathrm{fu}}=3.168>2.927=\omega_{\phi \varepsilon},
$$

is indicative of divergence, Fig 8.

Figure 7 demonstrates the convergence of stress 


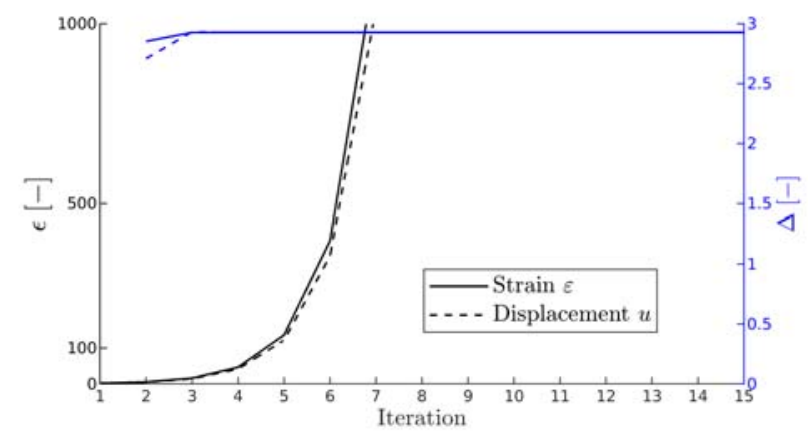

Figure 8: Strain and displacement in the iterative flexibility cell context. Difference quotient $\Delta$ at constant level, augmenting error $\epsilon$.

and force in the flexibility context. Figure 8 refers to the divergent behaviour of the kinematic quantities, strain and displacement. In either case the difference quotient of sequential iterates is seen close to the theoretical convergence indicator.

\subsection{The stiffness procedure}

\subsubsection{Strain and stress}

The iteration for the strain at given stress refers to eqn (25). Actually,

$$
\begin{aligned}
{\left[\begin{array}{c}
\varepsilon_{\mathrm{t}}^{\alpha} \\
\varepsilon_{\mathrm{t}}^{\beta} \\
\varepsilon_{\mathrm{t}}^{\gamma}
\end{array}\right]_{i+1}=\left[\begin{array}{c}
2 \\
-\frac{4}{3} \\
-\frac{4}{5}
\end{array}\right] } \\
-\left[\begin{array}{ccc}
0 & -\frac{3}{4} & -\frac{1}{4} \\
-\frac{2}{3} & 0 & \frac{1}{3} \\
-\frac{2}{5} & \frac{3}{5} & 0
\end{array}\right]\left[\begin{array}{c}
\varepsilon_{\mathrm{t}}^{\alpha} \\
\varepsilon_{\mathrm{t}}^{\beta} \\
\varepsilon_{\mathrm{t}}^{\gamma}
\end{array}\right]_{i} .
\end{aligned}
$$

The spectral norm of the operator matrix

$$
\omega_{\kappa \varepsilon}=1.0625
$$

affects convergence:

$$
\begin{aligned}
\varepsilon_{\mathrm{t}, 15} & =\left\{\begin{array}{lrr}
2.721 & -0.734 & -0.725
\end{array}\right\} \\
\varepsilon_{\mathrm{t}} & =\left\{\begin{array}{lll}
2.000 & 0.000 & 0.000
\end{array} .\right.
\end{aligned}
$$

The stress at fixed strain is approached by the scheme of eqn (29) as

$$
\begin{aligned}
{\left[\begin{array}{l}
\sigma_{\mathrm{c}}^{\alpha} \\
\sigma_{\mathrm{c}}^{\beta} \\
\sigma_{\mathrm{tc}}^{\gamma}
\end{array}\right]_{i+1}=\left[\begin{array}{l}
4 \\
0 \\
0
\end{array}\right] } \\
+\left[\begin{array}{ccc}
-1 & -\frac{4}{3} & 0 \\
-\frac{3}{2} & -\frac{5}{4} & \frac{3}{4} \\
0 & \frac{5}{12} & -\frac{1}{4}
\end{array}\right]\left[\begin{array}{c}
\sigma_{\mathrm{c}}^{\alpha} \\
\sigma_{\mathrm{c}}^{\beta} \\
\sigma_{\mathrm{c}}^{\gamma}
\end{array}\right]_{i}
\end{aligned}
$$

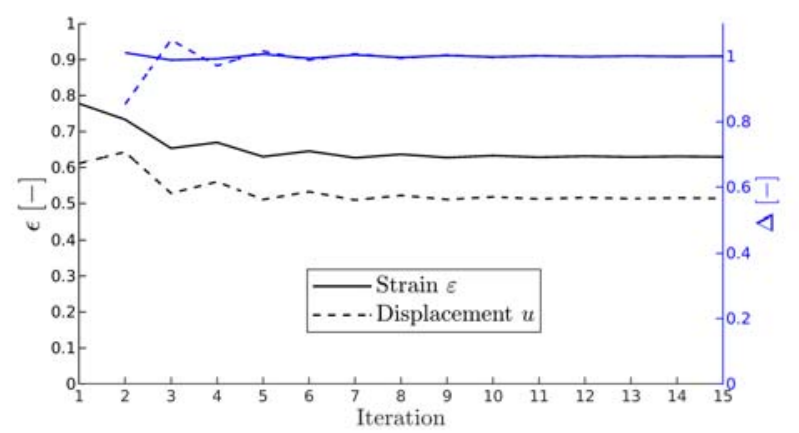

Figure 9: Strain and displacement in the iterative stiffness cell context. Difference quotient $\Delta$ and nondiminishing error $\epsilon$.

The spectral norm of the operator matrix

$$
\omega_{\kappa \sigma}=2.6387 \text {, }
$$

excludes convergence.

\subsubsection{Displacement and force}

The scheme of eqn (12) is implemented for the displacement at given force

$$
\begin{aligned}
{\left[\begin{array}{c}
u_{\mathrm{t}}^{\alpha} \\
u_{\mathrm{t}}^{\beta} \\
u_{\mathrm{t}}^{\gamma}
\end{array}\right]_{i+1}=\left[\begin{array}{c}
2 \\
-\frac{2 \sqrt{3}}{3} \\
-\frac{2}{5}
\end{array}\right] } & (110) \\
- & {\left[\begin{array}{ccc}
0 & -\frac{\sqrt{3}}{2} & -\frac{1}{2} \\
-\frac{\sqrt{3}}{3} & 0 & \frac{\sqrt{3}}{3} \\
-\frac{1}{5} & \frac{\sqrt{3}}{5} & 0
\end{array}\right]\left[\begin{array}{c}
u_{\mathrm{t}}^{\alpha} \\
u_{\mathrm{t}}^{\beta} \\
u_{\mathrm{t}}^{\gamma}
\end{array}\right]_{i} }
\end{aligned}
$$

The spectral norm of the operator matrix

$$
\omega_{\mathrm{ku}}=1.1219>1.0625=\omega_{\kappa \varepsilon},
$$

is higher but not far from that of the material level. Similarly, convergence is affected:

$$
\begin{aligned}
\mathbf{u}_{\mathrm{t}, 15}=\left\{\begin{array}{lll}
2.721 & 0.636 & 0.3625
\end{array}\right\} \\
\mathbf{u}_{\mathrm{t}}=\left\{\begin{array}{lll}
2.000 & 0.000 & 0.0000
\end{array}\right\}
\end{aligned}
$$

The solution for the force at fixed displacement is by the scheme of eqn (18)

$$
\begin{aligned}
{\left[\begin{array}{c}
S_{\mathrm{c}}^{\alpha} \\
S_{\mathrm{c}}^{\beta} \\
S_{\mathrm{c}}^{\gamma}
\end{array}\right]_{i+1}=\left[\begin{array}{l}
4 \\
0 \\
0
\end{array}\right] } & (111) \\
& -\left[\begin{array}{ccc}
-1 & -\frac{2 \sqrt{3}}{3} & 0 \\
-\sqrt{3} & -\frac{5}{4} & \frac{\sqrt{3}}{4} \\
0 & \frac{5 \sqrt{3}}{12} & -\frac{1}{4}
\end{array}\right]\left[\begin{array}{c}
S_{\mathrm{c}}^{\alpha} \\
S_{\mathrm{c}}^{\beta} \\
S_{\mathrm{c}}^{\gamma}
\end{array}\right]_{i} .
\end{aligned}
$$




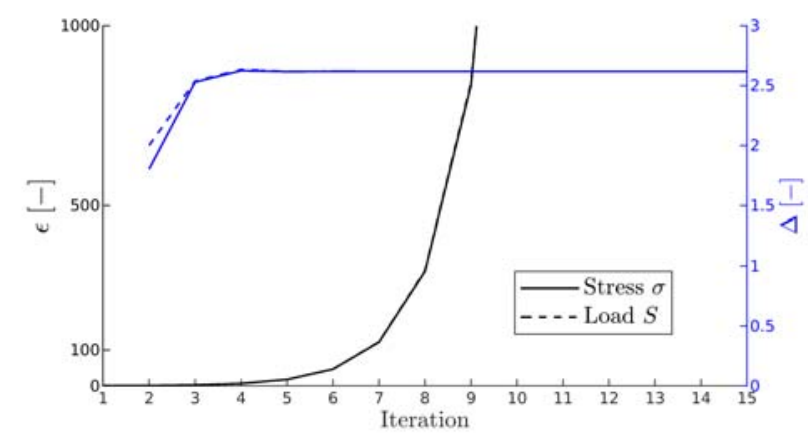

Figure 10: Stress and force in the iterative stiffness cell context. Difference quotient $\Delta$ and augmenting error $\epsilon$.

In the above procedure the indicator

$$
\omega_{\mathrm{kS}}=2.683 \text {. }
$$

predicts divergence.

Figure 9 shows a non-divergent behaviour for strain and displacement in the stiffness context, and the quotient of sequential iterates to be close to the convergence criterion. Figure 10 refers to the divergent behaviour of the static quantities, stress and force.

\section{The discretized structure}

The finite element equations establish the equilibrium between the stress resultants at the nodal points of the discretization mesh arranged in the block-vector array $\mathbf{S}=\left\{\mathbf{S}_{n}\right\}$ and the forces $\mathbf{P}=\left\{\mathbf{P}_{n}\right\}$ acting at the same positions. The single vectors $\mathbf{S}_{n}$, $\mathbf{P}_{n}, n=1, \cdots, n o p$, at the nop nodal points are defined by their components in a unique Cartesian reference system. The natural displacements of each of the nel elements in $\left\{\mathbf{u}_{\mathrm{te}}\right\}, e=1, \cdots$, nel derive from the displacements $\mathbf{u}=\left\{\mathbf{u}_{n}\right\}$ of the mesh nodal points. Symbolically by the matrix operation

$$
\left\{\mathbf{u}_{\mathrm{te}}\right\}=\mathbf{a}_{\mathrm{t}} \mathbf{u} .
$$

Virtual work equality relates the stress resultants in the mesh to those of the elements collected in the vector array $\left\{\mathbf{S}_{\mathrm{c} e}\right\}$ :

$$
\mathbf{S}=\mathbf{a}_{\mathrm{t}}^{\mathrm{t}}\left\{\mathbf{S}_{\mathrm{ce}}\right\} .
$$

The stress resultants $\mathbf{S}_{\mathrm{ce}}$ of the single element are expressed in terms of the displacements by the partitioned eqn (5) along with eqn (7). Using eqn (112) for the kinematics

$$
\mathbf{S}=\left(\mathbf{K}_{\mathrm{D}}+\mathbf{K}_{-}\right) \mathbf{u},
$$

where the structural matrices are composed of the elemental ones as

$$
\begin{array}{r}
\mathbf{K}_{\mathrm{D}}=\mathbf{a}_{\mathrm{t}}^{\mathrm{t}}\left\lceil\mathbf{k}_{\mathrm{De}}\right\rfloor \mathbf{a}_{\mathrm{t}}, \quad \mathbf{K}_{-}=\mathbf{a}_{\mathrm{t}}^{\mathrm{t}}\left\lceil\mathbf{k}_{-e}\right\rfloor \mathbf{a}_{\mathrm{t}} \\
\mathbf{K}_{-} \mathbf{u}=\mathbf{a}_{\mathrm{t}}^{\mathrm{t}}\left\{\left(\mathbf{k}_{-} \mathbf{u}_{\mathrm{t}}\right)_{e}\right\}=\mathbf{J}_{\mathrm{k}} .
\end{array}
$$

The matrix $\mathbf{K}_{\mathrm{D}}$ pertains to the cell assembly and $\mathbf{K}_{-}$ is the complement to the finite element representation of the structure. The diagonal matrix $\left\lceil\mathbf{k}_{\mathrm{D} e}\right\rfloor$ extends over all elements as does the block-diagonal matrix $\left\lceil\mathbf{k}_{-e}\right\rfloor$. The vector $\mathbf{J}_{\mathbf{k}}=\left\{\left(\mathbf{k}_{-} \mathbf{u}_{\mathrm{t}}\right)_{e}\right\}$ is set up with element contributions, while the matrix $\mathbf{K}_{-}$is not effective but serves the formal statement of the iteration scheme.

Equating the nodal stress resultants from eqn (114) with the applied forces and distinguishing the spring cell assembly, establishes an iterative solution scheme for the displacements founded on the partitioned stiffness method. Formally,

$$
\mathbf{K}_{\mathrm{D}} \mathbf{u}_{i+1}=\mathbf{P}-\mathbf{K}_{-} \mathbf{u}_{i},
$$

Then for the propagation of differences between consecutive iteration cycles by the stiffness cell,

$$
\mathbf{K}_{\mathrm{D}}(\delta \mathbf{u})_{i+1}=-\mathbf{K}_{-}(\delta \mathbf{u})_{i} .
$$

Tracing back to the constituing elements allows the statement of a sufficient condition for convergence. To this end the assembly in eqn (117) is considered resolved

$$
\mathbf{a}_{\mathrm{t}}^{\mathrm{t}}\left\lceil\mathbf{k}_{\mathrm{D} e}\right\rfloor\left\{\delta \mathbf{u}_{\mathrm{t} e}\right\}_{i+1}=-\mathbf{a}_{\mathrm{t}}^{\mathrm{t}}\left\lceil\mathbf{k}_{-e}\right\rfloor\left\{\delta \mathbf{u}_{\mathrm{t} e}\right\}_{i}
$$

The equation is fulfilled if

$$
\begin{array}{r}
\mathbf{k}_{\mathrm{D} e}\left(\delta \mathbf{u}_{\mathrm{t} e}\right)_{i+1}=-\mathbf{k}_{-e}\left(\delta \mathbf{u}_{\mathrm{t} e}\right)_{i} \\
e=1, \cdots, n e l,
\end{array}
$$

which points on eqn (13) for the individual element and reveals the requirement of eqn (16) for all elements as a sufficient condition for convergence of the assembly.

In the flexibility cell method the forces $\mathbf{S}_{\mathrm{ce}}$ for the single element are obtained in terms of the displacements $\mathbf{u}_{\mathrm{te}}$ by eqn (9) in conjunction with eqn (36)

$$
\begin{array}{r}
\mathbf{S}_{\mathrm{c} e}=\left(\mathbf{f}_{\mathrm{D}}^{-1}-\mathbf{f}_{\mathrm{D}}^{-1} \mathbf{f}_{-} \mathbf{f}_{\mathrm{N}}^{-1}\right)_{e} \mathbf{u}_{\mathrm{te}} \\
=\left(\tilde{\mathbf{k}}_{\mathrm{D}}+\tilde{\mathbf{k}}_{-}\right)_{e} \mathbf{u}_{\mathrm{te}} .
\end{array}
$$

The notation in the last expression stands for

$$
\begin{array}{r}
\tilde{\mathbf{k}}_{\mathrm{D} e}=\mathbf{f}_{\mathrm{D} e}^{-1} \\
\tilde{\mathbf{k}}_{-e}=-\left(\mathbf{f}_{\mathrm{D}}^{-1} \mathbf{f}_{-} \mathbf{f}_{\mathrm{N}}^{-1}\right)_{e}=\mathbf{f}_{\mathrm{N} e}^{-1}-\mathbf{f}_{\mathrm{D} e}^{-1},
\end{array}
$$


which identifies $\tilde{\mathbf{k}}_{\mathrm{De}}$ as the inverse diagonal of the element flexibility matrix, and $\tilde{\mathbf{k}}_{-e}$ as the element stiffness matrix diminished by the inverse diagonal of the flexibility matrix.

Assembling for the discretized structure,

$$
\mathbf{S}=\left(\tilde{\mathbf{K}}_{\mathrm{D}}+\tilde{\mathbf{K}}_{-}\right) \mathbf{u}
$$

which is formally as in eqn (114) but with the structural matrices composed of those elemental ones that are defined in eqn (121). The matrix $\tilde{\mathbf{K}}_{\mathrm{D}}$ pertains to the assembly of the flexibility based cells and $\tilde{\mathbf{K}}_{-}$is the complement to the finite element representation of the structure. Actually, the complementary terms in eqn (116) and eqn (122) enter the computation as load vectors assembled of element contributions avoiding matrix multiplication at the structural level.

The iteration scheme for the displacements

$$
\tilde{\mathbf{K}}_{\mathrm{D}} \mathbf{u}_{i+1}=\mathbf{P}-\tilde{\mathbf{K}}_{-} \mathbf{u}_{i},
$$

does not differ from the stiffness cell counterpart, eqn (116), but the system matrices pertaining to the present flexibility cell may alter the convergence behaviour. The propagation of differences between consecutive iteration cycles involves the individual properties of the discretized structure.

Application of the arguments that led to eqn (119) in the stiffness cell context to the iteration scheme of eqn (123) associated with the flexibility cell leads under consideration of eqn (121) elementwise to

$$
\begin{array}{r}
\left(\delta \mathbf{u}_{\mathrm{t} e}\right)_{i+1}=\mathbf{f}_{-e} \mathbf{f}_{\mathrm{N} e}^{-1}\left(\delta \mathbf{u}_{\mathrm{t} e}\right)_{i}, \\
\\
e=1, \cdots, \text { nel } .
\end{array}
$$

This points on eqn (38) for the individual element and reveals the requirement of eqn (40) for each element as a sufficient condition for convergence of the discretized structure. Since the finite element procedure implies a solution for the displacement, the flexibility cell participates via the secondary scheme of eqn (37) with convergence properties inferior to those of the stiffness cell, that enters the computation with the primary scheme of eqn (12).

For closer considerations with regard to the eigenvalue bounds of finite element matrices refer to the early works $[12,13]$ and to the more recent [14].

\section{Summary and conclusion}

Two defective spring cell models deriving from simplex finite elements are enriched to complete the substitution. For this purpose, the springs, or bars, which originally cover the diagonal entities of the flexibility matrix and for the stiffness matrix of the element are supplemented by displacements or forces, respectively, which account for the discarded off-diagonal entities of the element matrices. This produces forms that are implicit in the force for the flexibility approach, and implicit in the displacement for the stiffness approach. Apart from this direct, primary formalism, secondary schemes utilize the force-displacement relation of the element and viceversa in interchanging the implicit terms.

The spectral norm of the operator matrices defines the convergence indicators for an assessment of the iteration schemes ensuing in the case of prescribed force and of prescribed displacement. The procedures are explored in detail for triangular configurations. The rate of convergence decreases in departure from the ideal constellation of no off-diagonal entities (regular triangle, $\nu=1 / 3$ ). The range of convergent solutions is seen larger for the primary schemes. That is in terms of the force in the flexibility context, and in terms of the displacement in the stiffness counterpart. Accordingly, the flexibility concept appears favourable for an iterative solution of the stress, and the stiffness concept for an iterative solution of the displacement, with consequences for the treatment of the discretized structure. It is noted that The iteration procedures along with the theoretical arguments have been demonstrated with a numerical example. Thereby the relevance of the convergence indicators is confirmed as are the associated predictions.

The equation for the discretized structure as set up for the enriched spring cell substitutes of the finite element is inherently implicit. Since the problem is stated in terms of the displacement, the flexibility approach enters the iterative solution with the secondary, deduced form, while the stiffness approach participates directly with the primary form. A sufficient condition for convergence of the iteration process for the assembly requires convergence of each constituing cell inividually. Thereby iteration for completeness by the stiffness approach appears rather favourable than by the flexibility approach. It is recalled that the originally incomplete flexibility cell in terms of the elastic energy is closer to the finite element than its stiffness counterpart, however.

Acknowledgment: Maximilian Brodbeck carried out the computations and produced the graphics.

\section{References:}

[1] J. Argyris, Three-dimensional anisotropic and inhomogeneous elastic media - matrix analysis for small and large displacements, IngenieurArchiv 34, 1965, pp. 33-55. 
[2] I. Doltsinis, A note on the flexibility and stiffness natural approach to the triangular spring cell, Eng. Computat. 35, 2018, pp. 130-139.

[3] I. Doltsinis, From the natural continuum to the elastic spring cell in three and two dimensions - a flexibility approach, Eng. Computat. 10.1108/EC-11-2018-0543.

[4] T. S Marques, V. Dias da Silva and E. N. B. S. Jlio, Form finding of continua shells with lattice spring models, Eng. Struct. 10.1016/j.engstruct.2019.109683

[5] I. Doltsinis, M. Reck and V. Dias da Silva, Spring lattice models in the nonlinear analysis of membrane shells - an applicability study in elasticity, plasticity and damage, Comp. \& Struct. 10.1016/j.compstruc., 2019, 106174.

[6] I. Doltsinis, Stiffness vs flexibility based triangular spring cell - analysis of performance, Eng. Computat. 10-1108/EC-10-2019-0495.

[7] I. Doltsinis, Natural spring cell substitutes of simplex finite elements, WSEAS Transactions on Applied and Theoretical Mechanics 15, 2020, Art. 6, pp. 38-45. https://doi.org/10.37394/232011.2020.15.6

[8] R. Jarzabek, Einsatz, Bewertung und Entwickung von Stabgittermodellen mit einer Erweiterung auf die Wärmeleitung, Dissertation Universität Stuttgart, 2017.

[9] M. Reck, Lattice spring methods for arbitrary meshes in two and three dimensions, Intern. J. Numer. Methds. Engng. 2016, DOI 10.1002/nme.5358.

[10] M. Reck, Entwicklung kontinuumskompatibler Federmodelle, Dissertation Universität Stuttgart, 2017.

[11] M. Marcus, Basic Theorems in Matrix Theory, National Bureau of Standards, Appl. Math. Series $57,1960$.

[12] B.M. Irons, G. Treharne, A bound theorem in eigenvalues and its practical applications, Proceedings of the 3rd Conference on Matrix Methods in Structural Mechanics, Wright-Patterson AFB, Ohio, 1971, US Government Printing Office, Washington, DC, 1974, pp. 245-254.

[13] I. Fried, Bounds on the spectral and maximum norms of the finite element stiffness, flexibility and mass matrices, Internat.J. Solid Struct. 9, 1973, pp. 10131034.

[14] D. Loghina, M. van Gijzenb, E. Jonkers, Bounds on the eigenvalue range and on the field of values of non-Hermitian and indefinite finite element matrices, Journal of Computational and Applied Mathematics 189, 2006, pp. 304323.

\section{Creative Commons Attribution License 4.0} (Attribution 4.0 International, CC BY 4.0)

This article is published under the terms of the Creative Commons Attribution License 4.0

https://creativecommons.org/licenses/by/4.0/deed.en_US 